\section{OP61 DEVELOPMENT AND CERTIFICATION OF QUALITY PATIENT DECISION AIDS}

${ }^{1} S$ Schellinger*, ${ }^{2}$ B Hammes. 'Allina Health, St. Paul, MN, USA; ${ }^{2}$ Respecting Choices A Division of CTAC Innovations, La Crosse, WI, USA

10.1136/spcare-2019-ACPICONGRESSABS.61

Background Individuals with serious illness face complex healthcare decisions which have important and lasting consequences. Person-centered care can be achieved when personal values, goals, and beliefs are aligned with the actual care provided.

Person-centered decision-making requires a) clear, accurate and unbiased information about all options, including risks versus benefits; b) clinician investment and expertise in engaging and communicating with patients; and c) the effective integration of personal values, goals, and beliefs into choices. Person-centered decision aids (PDAs) are tools designed to help patients and providers in the process of shared decisionmaking. Research shows, high quality PDAs lead to increased knowledge, more accurate risk perception, reduced indecision about care, and improved patient engagement.

Methods Based on criteria developed by the International Patient Decision Aids Standards Collaboration (IPDAS), Healthier Washington Initiative, Washington State, USA, developed and implemented a process of certifying PDAs to assure they are effective, accurate, unbiased tools to use in the shared decision-making process. Implementation of quality PDAs can be standardized using the fundamentals described in the National Quality Forum's(NQF) National Quality Partners (NQP) Playbook-Shared Decision-Making in HealthCare.

Results This presentation will review certification criteria for developing high-quality PDAs. A four-phased approach will be discussed that describes the process of developing and certifying three serious illness decision aids and their integration within Respecting Choices ${ }^{\circledR}$ person-centered decision-making programs.

Conclusion Development and certification of quality PDAs in healthcare organizations using a standard approach to the decision-making process can support a culture of person-centered care.

\section{OP62 SUSTAINABLE IMPLEMENTATION OF ADVANCE CARE PLANNING IN ASIA: AN INTERPRETIVE-SYSTEMIC FRAMEWORK FOR NATIONAL DEVELOPMENT}

\begin{abstract}
${ }^{1,2} \mathrm{Ho}$ Ahy, ${ }^{2} \mathrm{P}$ Lall, ${ }^{2,3,4} \mathrm{WS}$ Tan, ${ }^{1,3} \mathrm{PV}$ Patinadan, ${ }^{3} \mathrm{LH}$ Wong, ${ }^{1} \mathrm{O}$ Dutta, ${ }^{5}$ WS Pang, ${ }^{6} \mathrm{CK}$ Low, ${ }^{7}$ E Zhuang, ${ }^{2,8} \mathrm{j}$ Car. ${ }^{1}$ School of Social Sciences, Nanyang Technological University, Singapore; ${ }^{2}$ Centre for Population Health Sciences, Lee Kong Chian School of Medicine, Nanyang Technological University, Singapore; ${ }^{3}$ NTU Institute for Health Technologies, Interdisciplinary Graduate School, Nanyang Technological University, Singapore; ${ }^{4}$ National Healthcare Group, Singapore; ${ }^{5}$ Lee Kong Chian School of Medicine, Nanyang Technological University, Singapore; ${ }^{6}$ Economics Programme, School of Social Sciences, Nanyang Technological University, Singapore; ${ }^{7}$ Agency for Integrated Care, Singapore; ${ }^{8} \mathrm{Global}$ eHealth Unit, School of Public Health, Imperial College London, UK
\end{abstract}

\subsection{6/spcare-2019-ACPICONGRESSABS.62}

Background To examine the underpinnings of Asia's first national Advance Care Planning (ACP) programme, and to identify the dynamics, mechanisms and systemic factors that influence the implementation of ACP in Singapore.

Methods A qualitative interpretive-systemic focus group study with 4 professional stakeholder groups who played critical roles in the ACP programme. Study included 63 physicians, nurses, medical social worker and allied health workers from 7 public hospitals and specialist center that incorporated ACP into clinical practice.

Results Framework analysis revealed 19 themes, organized into 5 categories including: (1) Life and Death Culture (social perception of death, biomedical model, health system hierarchy, health seeking behaviors), (2) ACP Coordination (institutional leadership, programme receptiveness, interdisciplinary trust, preparatory training), (3) ACP Administration (practice diversity, work flow, operation clarity), (4) ACP Outcomes (care preferences, medical-social dissonance, performance measures, intrinsic values), and (5) Sustainability Shift (public life and death education, holistic end-of-life care training, governance and service alignment, empowered citizenry). These further formed an interpretive-systemic framework of sustainable ACP, reflecting the social, cultural, political, operational and spiritual contexts that support national ACP development.

Conclusion This research provides insights on developmental and implementation challenges of Asia's first national ACP programme. ACP should be supported by public health strategy for enhancing individual, professional, and institutional readiness for end-of-life conversation before programme commencement. It emphasizes the importance of health policy, organizational structure, social discourse, and shared meaning in planning and delivery of ACP to aid care decision making among Asian patients and their families facing terminal illness and mortality.

\section{OP63 ESTIMATING AND COMMUNICATING PROGNOSIS IN PALLIATIVE CARE: A CROSS-SECTIONAL SURVEY AMONG PHYSICIANS IN THE SOUTHWEST REGION OF THE NETHERLANDS}

M Engel* ${ }^{*}$ A Van der Ark, R Tamerus, A van der Heide. Erasmus MC, University Medical Center Rotterdam, Rotterdam, Netherlands

\subsection{6/spcare-2019-ACPICONGRESSABS.63}

Background Advance care planning is important for patients with an advanced illness and a limited life expectancy. We explored experiences from physicians from different settings with estimating and communicating patients' poor prognosis. Methods A survey study was performed in 2017 in the Southwest region of the Netherlands among a random sample of physicians working in primary care, hospitals and nursing homes $(n=2212)$.

Results 547 physicians participated: 259 general practitioners (GP's), 205 hospital physicians (HP's) en 83 nursing home physicians (NHP's). In total, 61.1\% stated that they can adequately estimate if a patient will die within a year; $66.7 \%$ stated that they can adequately estimate a life expectancy of less than three months and $76.1 \%$ a life expectancy of less than a week. When a patient is estimated to have a prognosis of less than one year, $75.0 \%$ of all physicians indicate that they always/often discuss their wishes for treatment and care. For patients with an estimated prognosis of less than three months, $85.9 \%$ of HP's discuss patients' wishes, compared to $96.1 \%$ of GP's and $91.6 \%$ of NHP's. After hospital admission of patients with a limited life expectancy, $29.0 \%$ of GP's and $16.9 \%$ of NHP's indicate that they always/often receive adequate information from HP's about patients' wishes. 